\title{
Medición de la Sensibilidad del Adulto con Niños de 6 a 36 Meses de Edad: Construcción y Análisis Preliminares de la Escala de Sensibilidad del Adulto, E.S.A.
}

\section{Adult Sensitivity Assessment in Interaction with 6-36 Months old Children: Design and Preliminary Analyses of Adult Sensitivity Scale}

\author{
María Pía Santelices \\ Claudia Carvacho \\ Chamarrita Farkas \\ Francisca León \\ Francisca Galleguillos \\ Erika Himmel \\ Pontificia Universidad Católica de Chile
}

(Rec: 04 de mayo de 2012 / Acept: 09 de agosto de 2012)

\begin{abstract}
Resumen
La sensibilidad del adulto ha sido ampliamente estudiada en la primera infancia, por asociarse a un vínculo de apego seguro con el niño(a). Existen diferentes instrumentos de medición de este constructo, principalmente a través de métodos observacionales. La mayoría de ellos requiere un contexto experimental o extensa capacitación. El objetivo del presente estudio es crear un nuevo instrumento para medir la sensibilidad en adultos significativos para el niño(a) de 6 a 36 meses de edad, de manera simple, denominado Escala de Sensibilidad del Adulto, E.S.A. Se evalúa una breve interacción de juego libre, la cual es filmada y codificada. A continuación se presenta el proceso de construcción del instrumento, sus propiedades psicométricas, el análisis de concordancia interjuez y la confiabilidad. Se analiza una muestra de 99 díadas adulto significativoniño(a) y se concluye que la E.S.A. presenta muy adecuadas propiedades psicométricas y puede ser utilizada en diferentes contextos familiar, educacional o instituciones.

Palabras clave: sensibilidad, primera infancia, psicometría, instrumentos.
\end{abstract}

\begin{abstract}
The adult sensitivity has been extensively studied in early childhood, for it is associated with a secure attachment bond with the child. Different sensitivity measuring instruments have been developed, primarily through observational methods. Most of them require extensive training or a complex experimental s setting. The aim of this study is to develop a new instrument to assess sensitivity in adult caretaker of children aged 6 to 36 months, with few requirements for its use. The context of measurement involved a brief free-play interaction, which is filmed and encoded. The instrument was applied to a sample of 99 significant adult-child dyads and the videos analyzed with a rubric for each sensitivity indicator. Results show that the instrument has adequate psychometric properties and can be used in different familiar, educational or institutional contexts. Keywords: sensitivity, early childhood, psychometrics and instruments.
\end{abstract}




\section{Introducción}

La sensibilidad del adulto significativo es un constructo que cobra relevancia en la teoría del apego, siendo considerada por algunos autores como un factor fundamental en la organización de éste (Ainsworth, Blehar, Waters, \& Wall, 1978). Bowlby (1969) sugiere que una de las condiciones que podría influir en el desarrollo del apego correspondería a la sensibilidad del adulto a las necesidades y señales del bebé, pues la confianza del niño en el acceso, disponibilidad y responsividad del adulto a sus comunicaciones, así como su capacidad para sostener su experiencia afectiva, son indispensable en la experiencia vincular (Bowlby, 1973). Cabe mencionar que en este contexto entenderemos por adulto a aquellos adultos significativos con quienes los niños pequeños interactúan diariamente la mayor parte del tiempo (Bowlby, 1969).

\section{Conceptualización de la sensibilidad del adulto}

Ainsworth y colaboradores (1978) definen sensibilidad como la habilidad de la madre para tomar conciencia, interpretar adecuadamente y responder de forma apropiada y contingente a las señales y comunicaciones del niño(a). Si bien este comportamiento se puede manifestar de diferentes maneras, en distintas situaciones y tipos de interacción, reflejaría una tendencia general de la madre a interactuar con el niño(a) de acuerdo a sus deseos y necesidades.

A partir de esta conceptualización, otros autores como Pederson, Moran, Sitko, Campbell, Ghesquire y Acton (1990) han profundizado y desarrollado el constructo, estableciendo que a los 12 meses del niño(a) la sensibilidad materna se relaciona tanto con la disponibilidad de ésta para responder a las señales y comunicaciones de su hijo(a), como a su reacción efectiva y contingente ante el estrés. A su vez, Birigen \& Robinson (1991, citado en Bretherton, 2000) postulan que la sensibilidad en la infancia implica una clara percepción y adecuada respuesta de la madre a las peticiones del niño(a), así como su habilidad para negociar en conflicto, ser cálida y tranquilizante, flexible, creativa y aceptar la individualidad de su hijo(a). Por otra parte, Van den Boom (1997) plantea que la sensibilidad está presente en toda conducta interactiva siempre y cuando el adulto sea capaz de guiar su conducta social, logrando promoverla en vez de interrumpir el intercambio. El autor destaca que a lo largo de las diferentes dimensiones de la parentalidad, la respuesta debiese ser adecuada, pronta y contingente. Finalmente cabe destacar la propuesta de Crittenden (2005), quien entiende la sensibilidad como un constructo diádico. Ésta correspondería a cualquier patrón de comportamiento del adulto que permite al niño(a) explorar una actividad con interés y espontaneidad y sin inhibiciones, exageraciones o afecto negativo. Siendo un adulto sensible aquel que puede llamar la atención del niño(a), lo mantiene involucrado y puede distinguir cuándo requiere ser estimulado o tranquilizado para que pueda focalizarse en la interacción.

\section{Estudios relacionados con la sensibilidad del adulto}

Uno de los primeros estudios fue realizado por Ainsworth y colaboradores (1978), quienes tras la medición realizada con la situación extraña (Ainsworth, \& Wittig, 1969), correlacionan la conducta de la madre en su hogar y los estilos de apego de sus respectivos hijos. Se evaluaron tanto conductas específicas de la madre como características generales de ésta en términos de sensibilidad materna. Los resultados dan cuenta que las madres de niños seguros tienden a ser más sensibles, es decir, muestran una sensibilidad a las señales y comunicaciones de sus hijos(as), una actitud cooperativa aceptando la autonomía de éstos, se aprecian accesibles y disponibles y emocionalmente expresivas. Por otra parte, los padres de niños inseguros ambivalentes son más insensibles y los padres de niños inseguros evitativos tienden a ser más rechazantes, poco expresivos, poco intrusivos y rígidos en el contacto.

$\mathrm{Al}$ igual que en el estudio de Ainsworth y colaboradores (1978), en la literatura es posible encontrar diversos estudios que dan cuenta de relaciones significativas entre sensibilidad materna y patrones de apego (Behrens, Parker, \& Haltigan, 2011; Mills-Koonce et al., 2007), incluso en estudios transculturales (Jin, Jacobvitz, Hazen, \& Jung, 2012). Siguiendo la conceptualización original de la sensibilidad, se ha logrado replicar los resultados obtenidos inicialmente, permitiendo así, dar cuenta de evidencia empírica de que padres responsivos y sensibles tienden a tener relaciones seguras con sus hijos a los 12 meses y padres insensibles o no responsivos tienen mayor riesgo de establecer vínculos inseguros (Belsky, 1999; De Wolff, \& Van IJzendoorn, 1997; Van IJzendoorn, 1995). Entre estos estudios se encuentran los de Belsky, Rovine y Taylor (1984); Grossman, Grossman, Spangler, Suess, y Unzner (1985, ambos citados en Cassidy, \& Shaver, 1999); así como en los estudios de Cox, Owen, Henderson y Margand (1992), e Isabella (1993).

Otro tipo de estudios que se han desarrollado en relación al apego, corresponden a aquellos que correlacionan la sensibilidad del adulto, medida con la escala original, con otras características de éste que pudieran estar asociadas al apego seguro. Por ejemplo, la sensibilidad materna se correlaciona con el mostrarse más involucrada con su bebé (Lyons-Ruth, Gonnel, Zoll, \& Stahl, citados en Isabella, 1993), con la pronta respuesta ante el estrés (Del Carmen, Pedersen, Huffman, \& Bryan, 1993, citados en Belsky, 1999), moderada y apropiada estimulación (Feldstein, Crown, Beebe, \& Jaffe, 1995 citados en Belsky, 1999; Tamis Le Monda, \& Bornstein, 2002, citados en Hirsh-Pasek, \& Burchinal, 2006), con calidez materna ante la expresión de señales positivas del niño(a) (Lohaus, Keller, Ball, Elben, \& Voelker, 2001), así como con afecto positivo, cálido e involucramiento 
(Isabella, 1993) y baja presencia de conductas hostiles (Lock, \& McMahon, 2006). Por el contrario, se ha observado que las reacciones emocionales negativas frente al llanto del hijo(a) y de otros infantes, se relacionan con una respuesta menos sensible frente a la angustia del hijo(a) (Leerkes, 2010 citado en Leerkes, Parade, \& Gudmundson, 2011).

Por otra parte, estudios que se basan en la definición original de la sensibilidad pero con nuevas escalas, también dan cuenta de una asociación entre una adecuada sensibilidad materna medida a los 4 meses de edad y apego seguro al año de vida (Braungart, Garwood, Powers, \& Wang, 2001). Behrens y colaboradores (2011) miden la sensibilidad a través de la aplicación de la escala Maternal Behavior Q-Set [MBQS] a la situación extraña (Ainsworth, \& Wittig, 1969), encontrando asociaciones significativas entre sensibilidad y conductas de reunión de los infantes, así como una diferenciación significativa entre las categorías de apego. En particular, se aprecia que las conductas sensibles de las madres de niños(as) con apego seguro e inseguro ambivalente no divergían mayormente, mientras que las conductas de las madres de niños(as) con apego inseguro evitativo son las que muestran diferencias significativas. Lo anterior se puede explicar ya que una variable de la sensibilidad corresponde a permitir el contacto o conductas de acercamiento, lo que se observa tanto en las madres de niños(as) con apego seguro como en las madres de niños(as) con apego inseguro ambivalente, pero en este último caso, de forma no consistente. A diferencia de lo que ocurre con niños(as) con apego evitativo, donde la madre falla en este tipo de conductas.

Finalmente, se han reportado estudios sobre apego y sensibilidad en adultos. Mills-Koonce et al. (2011) establecen que un estilo de apego adulto de tipo inseguro se relacionaría con conductas parentales menos sensibles, especialmente cuando altos niveles de estrés están presentes.

Cabe mencionar que no solo se ha estudiado la relación entre sensibilidad y patrones de apego, sino que también su relación con otras áreas del desarrollo. Uno de estos estudios corresponde a un reporte de NICHD Early Child Care Research Network (ECCRN) (2006), focalizado en el adulto fuera del hogar. Éste muestra que tras medir la cualidad de este ambiente en términos de cuidado positivo y sensible entre los 15 y 54 meses del niño(a), indica que alta cualidad del cuidado del niño(a) se relaciona con avances en el área cognitiva, lenguaje, así como mejor desarrollo socioemocional y relaciones con los pares. En esta misma línea, también existe evidencia de que el cuidado sensible y estimulante por parte de la madre, específicamente la expresión abierta de afecto, refuerzo físico y verbal, promueve el logro académico y el desarrollo social y cognitivo (Burchinal, Campbell, Bryant, Wask, \& Ramey, 1997; Shonkoff, \& Philips, 2000, citados en Hirsh-Pasek, \& Burchinal, 2006).
Hirsh-Pasek y Burchinal (2006) realizan un estudio longitudinal, en el cual concluyen que cuando la sensibilidad del adulto es sostenida y aumenta en el tiempo, medida desde los 6 meses a la edad de 6 años, promueve a la edad de 54 meses mejor rendimiento en lenguaje, alfabetización, competencias matemáticas y atención. Otros estudios han correlacionado la cualidad general de sensibilidad, cooperación, aceptación y responsividad de la madre con habilidades en el lenguaje (Tamis le Monda, \& Bornstein, 2002, citados en Hirsh-Pasek, \& Burchinal, 2006). Asimismo, Leigh (2011) establece que las interacciones maternas sensibles durante el primer año de vida, tuvieron un efecto positivo en el posterior desarrollo de habilidades de lenguaje expresivo a la edad de dos y tres años. Por su parte, Symons y Clark (2000) correlacionan la sensibilidad temprana de la madre medida a los 2 años del hijo(a) con habilidades posterior a la edad de 5 años en tareas de falsa creencia como expresión de la adquisición de la teoría de la mente en los niños(as).

Finalmente, desde el ámbito biológico se ha planteado como correlatos biológicos de la sensibilidad, tanto una relación genotípica asociada al transportador de serotonina (Mileva-Seitz et al., 2011), como cierta relación entre la reacción vagal de la madre, frente a la presencia de afectos negativos en su hijo (Mills-Koonce et al., 2007).

\section{Métodos de evaluación de la sensibilidad del adulto}

La sensibilidad ha sido medida en diversos contextos en interacciones videograbadas, tales como juego libre (Crittenden, 2005; NICHD ECCRN, 1997), visitas domiciliarias (Pederson, \& Moran, 1995) y la situación extraña (Ainsworth et al., 1978; Behrens et al., 2011).

En cuanto a los principales instrumentos, se encuentra inicialmente la Escala de Sensibilidad Materna de Ainsworth y colaboradores (1978). Ésta consiste en 4 escalas de 1 a 9 puntos que evalúan las siguientes dimensiones: sensibilidad - insensibilidad a las señales del bebé, aceptación - rechazo, cooperación - interferencia y accesibilidad - ignorancia. A cada una de éstas se asigna un puntaje dependiendo de descripción se ajusta la conducta observada de la madre.

Por otra parte, se creó la escala Maternal Behavior Q-Set [MBQS] a partir de la técnica de Q-Sort (Pederson, \& Morgan, 1995). En ésta se encuentra una alta predictibilidad con apego seguro y las escalas originales de Ainsworth. Específicamente, consta de un set de 90 descripciones de conductas operacionalizadas de interacción materna, que son clasificadas y comparadas con las de una madre sensible prototíipica, y se desarrolla en el contexto de una extensa visita domiciliaria (Behrens et al., 2011).

Por último, cabe mencionar que Crittenden (2005), quien como se menciona anteriormente conceptualiza sensibilidad como un constructo diádico, desarrolla un instrumento de medición considerando tal definición. Crea una versión para infantes de $0-15$ meses y para niños de $15-30$ meses, en 
éstas se evalúan siete aspectos de la conducta interaccional a través de frases descriptivas, otorgando categorías tanto al adulto como al niño. Con respecto al adulto, además de reportar niveles de sensibilidad, otorga dos categorías negativas distintas para el extremo de no-sensibilidad: controlador y no responsivo.

\section{Discusión respecto al constructo sensibilidad}

Si bien la sensibilidad ha sido considerada como el determinante primario de la calidad del apego a los 12 meses de edad (Crittenden, 2006), este planteamientos no ha estado exento de discusión en la comunidad académica. Específicamente, se ha cuestionado el real tamaño de efecto que puede tener la sensibilidad materna en el desarrollo del apego seguro, así como su rol mediador entre el estado del apego del adulto y del niño(a) (Atkinson et al., 2005). Goldsmith y Alansky (1978, citado en De Wolff, \& Van IJzendoorn, 1997) realizan el primer meta-análisis sobre apego y sensibilidad, concluyendo que si bien los estudios hasta la fecha han replicado el poder predictivo de la sensibilidad en el apego, el tamaño del efecto es mucho menor del pensado. Posteriormente, De Wolff y Van IJzendoorn (1997) publicaron un meta-análisis con 66 estudios que midieron sensibilidad materna y apego. El análisis de correlación de Pearson y la comparación con tamaños de efecto según los criterios de Cohen (1988), indicó un tamaño de efecto de la asociación entre dichas variables a un nivel bajo $(r=0.22, N$ $=1.666$ ), que solo aumentó a medio cuando se consideraron exclusivamente los trabajos que utilizaron las medidas de sensibilidad con la escala original de Ainsworth $(r=0.24$, $N=837)$. Los autores concluyen, a partir de estos datos, que se comprueba el rol de la sensibilidad en el desarrollo del apego como fue planteado por Bowlby (1969), pero que éste no puede ser considerado como el factor más importante para el desarrollo del apego (De Wolff, \& Van IJzendoorn, 1997; Raval et al., 2001 citado en Prior, \& Glaser, 2006).

En la misma línea, Thompson (1997) concluye que la sensibilidad es una rúbrica que incluye varias cualidades de las prácticas del adulto que pueden tener diversas consecuencias en la conducta del niño(a), por lo que algunas de dichas consecuencias no necesariamente sean relevantes para el apego.

Debido a las dificultades apreciadas para definir el constructo de sensibilidad, se ha complejizado identificar aquellos aspectos de la interacción materna que contribuyen directamente al apego seguro. Ante tal situación hay autores que han tratado de resolver este problema buscando cuáles son las conductas específicas y críticas de sensibilidad que correlacionan con apego seguro. El estudio desarrollado por Cassidy y colaboradores (2005, citado en Prior, \& Glaser, 2006) utiliza la conceptualización de Ainsworth sobre sensibilidad y clasifica a las madres como globalmente sensibles o insensibles. Crittenden (2006) postula que debe ser considerada como una cualidad diádica y no individual de la interacción y por lo mismo, toda operacionalización y evaluación de sensibilidad debe reconocer los procesos del desarrollo del niño(a), pues un adulto puede ser solo sensible a medida que es responsivo a las características únicas del infante. La autora destaca que para la evaluación de sensibilidad es importante considerar simultáneamente una escala global de sensibilidad, conductas significativas en la interacción que puedan dar luces sobre el patrón predominante, comparación con casos ejemplares de cada interacción evaluada y una puntuación por dimensión específica que se evalúa.

Finalmente, los autores Bornstein, Tamis-LeMonda, Chun-Shin Hahn y Haynes (2008) proponen que la sensibilidad no debe ser considerada un constructo monístico ni medido por ello en una escala global única, sino que, por el contrario, es un constructo multidimensional, modular, independiente y cambiante en el tiempo. A esto agregan que distintos tipos de responsividad se dan en momentos diferentes del desarrollo y componentes específicos de ésta se corresponden con habilidades específicas en los niños. Asimismo, Hirsh-Pasek y Burchinal (2006) plantean la necesidad de reflexionar en torno a la diferencia de medir la sensibilidad centrada como variable única, por lo que se podría medir una sola vez en el tiempo o centrada como una variable de la persona, y por tal, dinámica a lo largo del tiempo.

A lo largo de los temas expuestos es posible apreciar que el constructo de sensibilidad se caracteriza por tener diferentes conceptualizaciones y formas de medición, a lo que se suma la discusión en torno a su influencia en el desarrollo del apego, de lo cual se desprenden diferentes posturas para explicar la razón de esta situación, tales como la falta de especificidad de la definición original del constructo (Isabella, \& Belsky, 1991; Isabella, 1993; Lamb, Thompson, Gorden, Charnov, \& Estes, 1984; Meins, Fernyhough, Fradley, \& Tuckey, 2001). Por otra parte, dada la importancia que implica la sensibilidad del adulto para el desarrollo del niño, resulta necesario proponer un método de evaluación que considere conductas específicas que dan cuenta de la sensibilidad del adulto y que pueda ser aplicado y analizado de forma eficiente, es decir, que requiera una capacitación breve y que pueda ser aplicado en diferentes contextos y culturas. $\mathrm{Al}$ respecto, es relevante contar con un instrumento validado en el país de origen (Chile), pero que también pueda ser utilizado en otros países, recogiendo las especificidades de la cultura.

Por lo tanto, el objetivo del presente estudio es analizar el instrumento E.S.A en una muestra piloto para evaluar sus propiedades psicométricas. Este estudio contempla un análisis preliminar de la nueva escala, requiriéndose en una segunda etapa nuevos análisis más exhaustivos y con una muestra representativa y de mayor tamaño. 


\section{Método}

\section{Participantes}

La muestra fue intencionada y estuvo compuesta por 99 díadas adulto significativo-niño(a), de las cuales 51 están conformadas por el apoderado del niño(a) y 48 constituidas por el personal educativo (educadora o técnica) del jardín infantil al cual asiste el niño(a).

Las edades de los niños de la muestra fluctúan entre los 10 y 33 meses de edad (Media $=22.11$, D.T. $=9.227)$. Debido a que el proyecto de investigación en el cual se inserta el presente estudio es longitudinal y recoge información de los niños en dos rangos de edad, se procedió a aplicar los instrumentos piloto en esos dos grupos. Un grupo de 46 niños de rango inferior de edad, de 10-14 meses (Media $=$ $12.0, D . T .=1.294)$, $\mathrm{y}$ un grupo de 53 niños de rango superior de edad, de 28-33 meses (Media $=30.25$, D.T. $=1.818$ ).

Se incluyeron en el estudio 18 jardines infantiles, de los cuales 8 pertenecen a un contexto de nivel socioeconómico medio, es decir, son jardines privados que implican un costo para las familias y 10 a nivel socioeconómico bajo, correspondientes a jardines infantiles públicos, gratuitos y que atienden a una población con vulnerabilidad psicosocial. El $39,4 \%$ de la muestra son niñas y $60,6 \%$, varones.

\section{Instrumento}

\section{Construcción del instrumento para Evaluar la Sensibilidad del Adulto (E.S.A)}

El instrumento para evaluar la sensibilidad del adulto fue desarrollado a partir de la revisión bibliográfica del constructo sensibilidad, así como revisando los instrumentos existentes para medir esta dimensión. Se diseñaron los indicadores en un equipo de expertos en el tema, en el cual participaron psicólogos clínicos especialistas en infancia temprana, educadoras y expertos en metodología. El instrumento está orientado a evaluar la sensibilidad en adultos significativos en interacción con niños de 6 a 36 meses de edad. La sensibilidad se define como la "contingencia en responder a las señales y comunicaciones del niño(a) a modo de favorecer su interés y cooperación en el juego, en un clima de afecto positivo". El contexto de evaluación corresponde a una interacción de juego libre entre el adulto y el(la) niño(a). Se considera que el juego libre es un contexto adecuado para la evaluación de sensibilidad, debido a que corresponde a una actividad cotidiana tanto para el niño como para el adulto, lo cual permitiría evaluar la interacción en un contexto común para los participantes, permitiendo así que el adulto se desenvuelva y despliegue sus competencias tal como lo haría en la cotidianidad de la relación, posibilitando una evaluación más cercana a lo que ocurre en la díada.
Por otra parte, el juego corresponde al medio natural para la expresión del niño(a), permitiendo, al ser un ambiente cotidiano, que se relacione fácilmente con los juguetes y en consecuencia, jugar (Nickerson, 1973, citado en West, 2000).

De acuerdo a lo anterior, se propone que la evaluación de la sensibilidad del adulto en su interacción con niños y niñas de 6 a 36 meses, se divide en 2 formas según grupo etario. Ambas formas se diferencian por los set de juguetes que incluyen, los cuales serían adecuados para cada nivel de desarrollo.

\section{Diseño del set de juguetes}

Se procedió a diseñar dos sets de juguetes, con el propósito de que fueran atractivos para los niños(as) y que permitiesen el desarrollo de un juego interactivo. Se consideró que ambos set fueran adecuados para la edad de cada grupo y que pudiesen incentivar el involucramiento de los adultos a evaluar. Para tales fines, se discutió el material en un grupo de expertos, para luego consultar la propuesta con un grupo experto externo.

El set final de juego se compone de los siguientes elementos:

- Set de juguetes Forma A, 6 a 23 meses: cubos, cascabel o maraca, libros con imágenes, auto, una muñeca con una mamadera, tuto o frazada, oso de peluche y un juego de encaje.

- Set de juguetes Forma B, 24 a 36 meses: cubos (e.g. lego), instrumento musical (tambor, pandereta), libros con imágenes y textos cortos, auto, una muñeca y comida de plástico, frazada o manta, mamadera y animales de zoológico o granja.

\section{Situación de evaluación}

La situación de evaluación consiste en una interacción filmada de 5 minutos de juego libre, en la cual se invita a la díada a sentarse de la manera que les resulte más cómoda a ambos (frente a frente o uno al lado del otro, invitándolos a sacar los juguetes de la caja, siguiendo la siguiente consigna: "La(lo) invito a disfrutar de un rato libre de juego con (nombre del niño o niña). No hay algo especifico que deban realizar, solamente haga lo que siempre hace o juegue como siempre juega con el niño(a)".

\section{Construcción de los indicadores del instrumento}

Inicialmente se elaboraron 30 indicadores que abordaban distintos aspectos de la interacción sensible. Debido a que algunos de ellos apuntaban a la descripción de la conducta sensible a través de todo el juego y otros apuntaban a conductas más específicas que respondían a ciertas conductas de los niños, se procedió a separar los indicadores en dos categorías: aquellos que se podían observar durante la totalidad de la interacción y otros que aluden a conductas 
específicas según las señales del niño(a) que pueden o no estar presentes durante el juego.

Posteriormente se revisaron 3 videos en el equipo de expertos y se procedió a reducir el número de indicadores a 26. Esta versión de 26 indicadores fue piloteada con la muestra de 99 casos, tras lo cual se eliminaron aquellos que correspondían a conductas específicas de los niños(as), debido a que no fueron conductas observadas en una frecuencia esperable. Por lo tanto, la versión final quedó compuesta por 19 indicadores (se anexa el instrumento con los 19 indicadores, ver Figura 1).

\section{Construcción de rúbrica de evaluación}

El sistema de codificación de la E.S.A. se basa en el análisis de la interacción de juego entre el adulto y el niño(a), utilizando para ello una rúbrica de evaluación que contiene

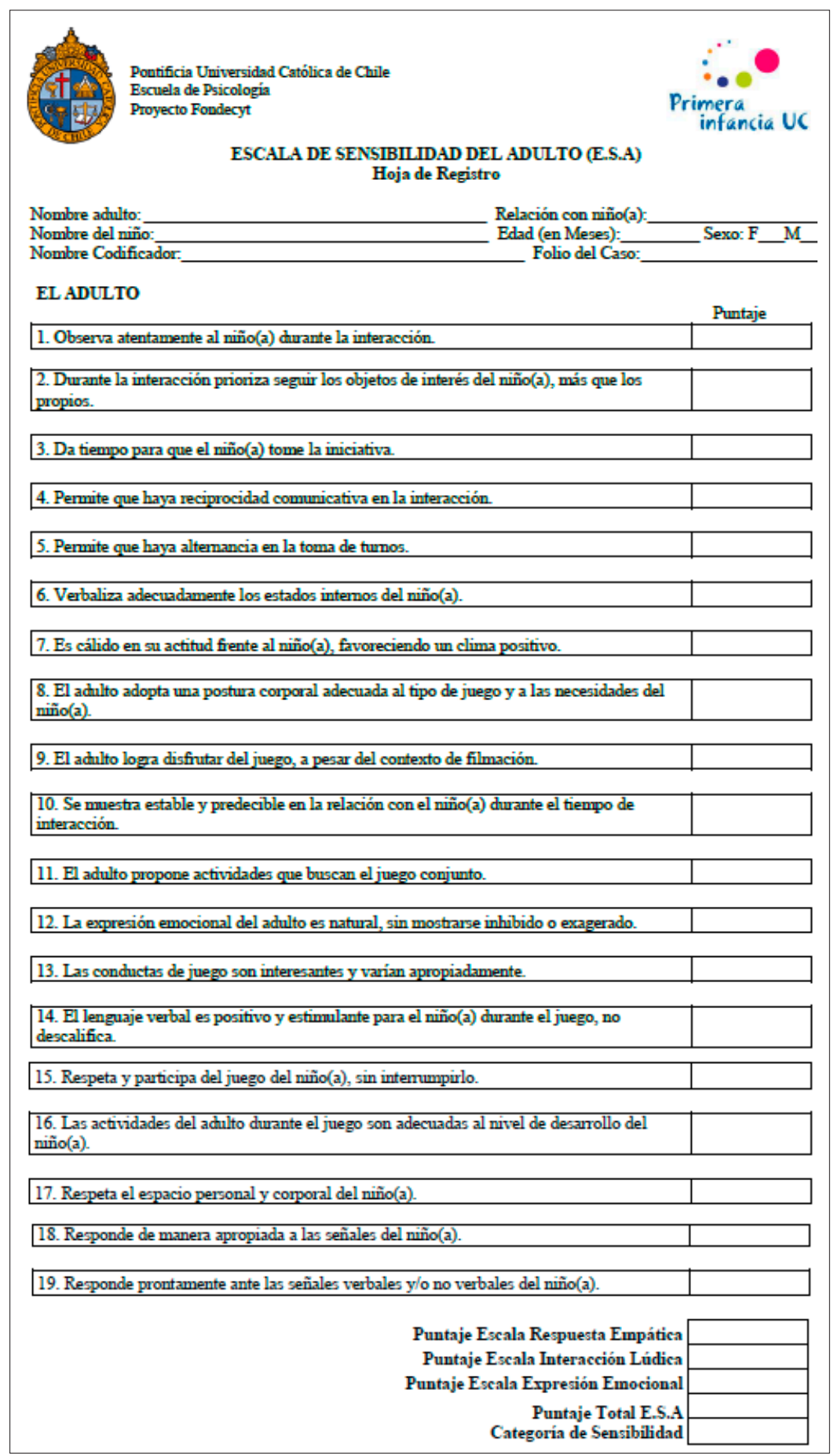

Figura 1. Instrumento E.S.A. 
una descripción detallada de la conducta que se asocia a los distintos puntajes.

Cada indicador se evalúa con puntajes 1, 2 ó 3, correspondientes a una baja, adecuada o alta sensibilidad, de modo que cada categoría respondiera a los niveles de sensibilidad.

La rúbrica de evaluación de la Escala de Sensibilidad del Adulto (E.S.A) tiene un diseño que le permite al codificador(a) tener en consideración, al momento de analizar la interacción, la conducta a evaluar, la definición de la misma (junto con ejemplos representativos) y una descripción exhaustiva de cada categoría de puntajes (1, 2 y 3$)$. Además, dispone de una hoja de registro para cada caso analizado, en la que se consigna el puntaje de cada ítem y un apartado de observaciones en caso de que sea necesario.

El sistema de codificación fue realizado por un equipo de 4 especialistas, el cual estuvo encargado del diseño del sistema y comprobación de su funcionamiento en casos reales a partir de videos, así como su supervisión y adaptación en cada etapa. Luego se envió el instrumento con su sistema de codificación a 3 jueces externos y se incorporaron sus sugerencias (se anexa un ejemplo de rúbrica de evaluación, ver Figura 2).

\section{Procedimiento}

\section{Procedimiento de recolección de datos}

Se contactó a jardines infantiles de financiamiento público y privado para la presentación del estudio. Una vez que el equipo educativo del centro accedió a participar del pilotaje del instrumento, se explicó a los potenciales participantes los objetivos y características del estudio, solicitándoles la firma de la carta de consentimiento informado.

Las filmaciones se realizaron en el establecimiento educacional, siempre que la participación del niño o niña fuese voluntaria. En todos los casos se contó con una sala con espacio e iluminación adecuados para la actividad. El aplicador(a) del instrumento fue previamente capacitado(a) y entrenado(a) por el equipo de investigación, con apoyo de un manual de aplicación diseñado para este fin.

\section{Procedimiento de evaluación de las interacciones}

La evaluación de los videos fue realizada por 2 expertos. Ambos fueron capacitados en el constructo y diseño de la propuesta de evaluación. Además, se realizaron 3 sesiones de entrenamiento en el sistema de evaluación y codificación.

Análisis de los datos:

Los análisis estadísticos están orientados a evaluar las propiedades métricas del instrumento en cuanto a concordancia interjueces y confiabilidad, a modo de evaluar la pertinencia de cada uno de los indicadores de la rúbrica y generar así, una propuesta definitiva. En primera instancia, se estableció el Coeficiente Kappa de Cohen (Cohen, 1960) para medir el grado de acuerdo entre los observadores para la propuesta inicial de 26 indicadores de la E.S.A. Para ello, 2 jueces entrenados codificaron de manera independiente 35 interacciones de juego seleccionadas al azar (35\% de la muestra total).

A partir de los resultados de la concordancia inter-jueces, se procedió a definir la versión final del instrumento compuesto por 19 indicadores y se analizó la confiabilidad a través del coeficiente Alfa de Cronbach. Además, se efectuaron los análisis de confiabilidad según sexo de los infantes, rol del adulto significativo, edad del niño(a) y NSE. También se analizó la correlación ítem-escala y su efecto sobre la estimación de confiabilidad.

Posteriormente se procedió a realizar un análisis factorial (método VARIMAX de componentes rotados), del cual se obtuvieron las 3 escalas del instrumento, así como la propuesta del cálculo del puntaje final y la asignación de la categoría correspondiente en sensibilidad (alta, adecuada o baja).

\section{Resultados}

\section{Análisis de la concordancia inter-jueces}

El análisis de concordancia se aplicó de forma diferenciada para aquellos ítems que son conductas que se pueden observar según señales específicas del niño(a) (indicadores

Ejemplo rúbrica de evaluación: ítem 11

2

11. El adulto propone acti- La mayoría de los juegos que propone vidades que buscan el juego el adulto no permiten que exista un conjunto. juego conjunto entre él y el niño(a). Ó si hay juego compartido, éste es esporádico y no representa el tipo de interacción.

El adulto algunas veces propone La mayoría de los juegos projuegos que buscan la participación puestos por el adulto permiten conjunta, mientras que en otros que exista un juego conjunto momentos no lo hace. y buscan la participación del niño(a), incluyéndolo.

Descripción: El adulto propone o realiza juegos cuyo objetivo es "jugar con el niño(a)" y no simplemente mostrar/pasarle juguetes o invitarle a jugar solo, sin involucrarse. La mayor parte de los juegos propuestos por el adulto son apropiados para que el niño(a) los comparta y lo incorporan (al adulto), siendo realizados de manera conjunta.

Figura 2. Ejemplo de rúbrica. 
Tabla 1. Coeficiente Kappa para los indicadores 1-19.

\begin{tabular}{cccccc}
\hline $\mathrm{N}^{\circ}$ Ítem & Kappa Observado & Error Estándar & $\mathrm{N}^{\circ}$ Ítem & Kappa Observado & Error Estándar \\
\hline 1 & .7797 & .1896 & 10 & .4204 & .2337 \\
2 & .6211 & .1609 & 11 & .72 & .1385 \\
3 & .6867 & .1608 & 12 & .9072 & .1834 \\
4 & .7569 & .1171 & 13 & .8402 & .1478 \\
5 & .6998 & .1717 & 14 & .5455 & .2118 \\
6 & .7172 & .1852 & 15 & .6295 & .1976 \\
7 & .5774 & .2183 & 16 & .5455 & .2545 \\
8 & .9347 & .1005 & 17 & .5165 & .2194 \\
9 & .6478 & .1932 & 18 & .8214 & .22 \\
& & & 19 & .8424 & .0791 \\
\hline
\end{tabular}

Nota. N: 35 casos, correspondientes al 35\% de la muestra.

$\mathrm{N}^{\circ} 1$ al $\left.\mathrm{N}^{\circ} 19\right)$; se observan durante toda la interacción y que incluyen 3 categorías de evaluación, de aquellos indicadores en los que solo se evaluó presencia o ausencia del indicador e incluyen categoría "No Aplica" (del ítem №20 al N²6). Para el primer conjunto se aplicó el Kappa ponderado y para el segundo se determinó el coeficiente Kappa considerando los acuerdos y diferencias de los jueces respecto a la presencia/ausencia del indicador en la interacción, mediante la Kappa lineal.

Los resultados de este análisis muestran que los evaluadores tuvieron un nivel de consistencia adecuado en la asignación de las categorías de las rúbricas para los diferentes indicadores generales. Este resultado constituye una evidencia para postular que las rúbricas son suficientemente explícitas para lograr evaluaciones concordantes de la sensibilidad, efectuada por observadores diferentes (ver Tabla 1).

En relación a los indicadores específicos a la conducta observada, dos de ellos no obtuvieron acuerdo en relación a su presencia o ausencia, y los indicadores restantes mostraron bajo acuerdo en el puntaje asignado al indicador cuando éste se encontraba presente. Al revisar en detalle los puntajes se pudo constatar que estos bajos resultados se debían a la baja frecuencia de presencia de estas conductas, por lo cual se optó por eliminar estos indicadores en la versión final.

En síntesis, los 19 indicadores conservados poseen suficiente acuerdo interjueces, ya que obtienen un nivel de acuerdo "aceptable", es decir, igual o mayor a .40 (Landis, $\&$ Coch, 1977).

\section{Análisis de la confiabilidad del instrumento}

Posteriormente, se analizó la confiabilidad para la versión definitiva del instrumento completo que, como se mencionó anteriormente, considera 19 indicadores. El coeficiente alpha que se obtuvo fue de 0.930 .

Adicionalmente, se analizó la confiabilidad del instrumento según características sociodemográficas de la muestra en cuanto a sexo del niño(a), grupo de edad, adulto evaluado (apoderado o personal educativo) y
NSE. Los resultados no presentan diferencias por grupo, manteniéndose una alta confiabilidad en cada caso. Según sexo del niño(a), la confiabilidad de los niños es 0.905 y la de las niñas es 0.923 . Respecto al grupo de edad, el tramo conformado por niños(as) entre 10 a 14 meses obtiene un Alpha de 0.915 y el tramo de 28 a 33 meses, 0.909. Respecto al adulto evaluado, la confiabilidad del grupo conformado por familiares es 0.892 y para el personal educativo es 0.929 . Finalmente, la distinción de NSE bajo y medio muestra una confiabilidad de $0.914 \mathrm{y}$ 0.911 , respectivamente.

De acuerdo a estos resultados, el instrumento no presentaría sesgos según sexo del niño(a), grupo de edad del niño(a), adulto evaluado y NSE, pero es necesario apreciarlos con cautela por el tamaño muestral en las categorías de análisis.

\section{Análisis de correlación ítem-escala}

Se examinaron las distribuciones de las puntuaciones de los ítems, así como su desviación estándar y la correlación ítem-escala. Las distribuciones ${ }^{1}$ resultan adecuadas, ya que en la mayor parte de los ítems las tres puntuaciones presentan frecuencias con porcentajes distribuidos en todas las categorías. Por otra parte, las correlaciones ítem-escala tienen valores iguales o superiores a .30 , lo cual indica que la escala es consistente en la medición del constructo que se pretende medir. La siguiente tabla contiene la información detallada por ítem.

En síntesis, de acuerdo a estos resultados, en su mayoría los ítems de la rúbrica de evaluación de la E.S.A. poseen una buena correlación con la escala total, pero se sugiere revisar a futuro la rúbrica para el ítem $\mathrm{N}^{\circ} 19$.

\section{Análisis factorial exploratorio}

Los 19 indicadores generales de la versión depurada del instrumento se sometieron a un análisis exploratorio

\footnotetext{
Las tablas referentes a las distribuciones no se incluyen aquí, por razones de espacio.
} 
Tabla 2. Análisis de correlación item-escala.

\begin{tabular}{lccccccc}
\hline Ítem & $\begin{array}{c}\text { Media } \\
\text { Ítem }\end{array}$ & $\begin{array}{c}\text { Desviación estándar } \\
\text { ítem }\end{array}$ & $\begin{array}{c}\text { Correlación } \\
\text { ítem-escala }\end{array}$ & Reactivo & $\begin{array}{c}\text { Media } \\
\text { Ítem }\end{array}$ & $\begin{array}{c}\text { Desviación estándar } \\
\text { ítem }\end{array}$ & $\begin{array}{c}\text { Correlación } \\
\text { ítem-escala }\end{array}$ \\
\hline 1 & 2.152 & 512 & .51 & 13 & 2.182 & .492 & .58 \\
2 & 1.980 & .585 & .81 & 14 & 2.071 & .369 & .60 \\
3 & 1.919 & .559 & .67 & 15 & 2.010 & .454 & .76 \\
4 & 1.859 & .546 & .77 & 16 & 2.212 & .329 & .49 \\
5 & 2.202 & .505 & .52 & 17 & 1.859 & .525 & .59 \\
6 & 2.253 & .512 & .60 & 18 & 2.354 & .390 & .62 \\
7 & 2.071 & .369 & .56 & 19 & 2.111 & .584 & .29 \\
8 & 1.980 & .565 & .42 & 20 & 2.141 & .546 & .73 \\
9 & 2.172 & .425 & .63 & 21 & 2.182 & .432 & .50 \\
10 & 2.162 & .297 & .37 & 22 & 2.222 & .375 & .68 \\
11 & 2.152 & .533 & .54 & 23 & 2.141 & .303 & .78 \\
12 & 2.606 & .259 & .34 & & & & .50 \\
\hline
\end{tabular}

Tabla 3. Análisis de componentes principales.

\begin{tabular}{|c|c|c|c|}
\hline \multirow{2}{*}{ Indicadores } & \multicolumn{3}{|c|}{ Componente } \\
\hline & 1 & 2 & 3 \\
\hline 1. Observa al niño(a) atentamente durante la interacción. & .367 & & \\
\hline 2. Prioriza seguir los objetos de interés del niño(a). & .786 & & \\
\hline 3. Da tiempo para que el niño(a) tome la iniciativa. & .818 & & \\
\hline 4. Permite que haya reciprocidad en la comunicación. & & .540 & \\
\hline 5. Permite que haya alternancia en la toma de turnos. & & .748 & \\
\hline 6. Verbaliza adecuadamente los estados internos del niño(a). & & & .789 \\
\hline 7. Es cálido en su actitud el niño(a), favoreciendo un clima positivo. & & & .394 \\
\hline 8. Adopta una postura corporal adecuada al tipo de juego. & & & .287 \\
\hline 9. Disfruta del juego pese al contexto de filmación. & & .551 & \\
\hline 10. Se muestra estable en la relación con el niño(a) durante la interacción. & & .542 & \\
\hline 11. Propone juegos que incorporan al niño(a). & & .790 & \\
\hline 12. Tiene una expresión emocional natural, sin mostrarse inhibido o exagerado. & & & .511 \\
\hline 13. Realiza un juego interactivo, interesante y variando apropiadamente. & & .587 & \\
\hline 14. Usa lenguaje positivo y estimulante para el niño(a) durante el juego, no descalifica. & & & .685 \\
\hline 15. Respeta el juego del niño(a) sin interrumpirlo. & .842 & & \\
\hline 16. Efectúa actividades adecuadas al desarrollo del niño(a). & & .434 & \\
\hline 17. Respeta el espacio personal y corporal del niño(a). & .416 & & \\
\hline 18. Responde de manera apropiada a las señales del niño(a). & .664 & & \\
\hline 19. Responde prontamente a las señales verbales o no verbales. & .681 & & \\
\hline
\end{tabular}

Método de extracción: Análisis de componentes principales.

Método de rotación: Normalización Varimax con Kaiser.

Matriz de componentes rotados, la rotación ha convergido en 5 iteraciones.

de componentes principales que mostró KMO de 0.822 , con un porcentaje de varianza común de $51,43 \%$ con 3 factores correspondientes a 20,23\%, 18,55\% y 12,65\%, respectivamente. Los ítems en los tres factores presentan pesos factoriales superiores a 0.25 (Tabla 3), lo que muestra que son suficientemente robustos. También puede destacarse que los indicadores se distribuyen bien entre los factores (7, en el primero; 6 , en el segundo y 6 en el tercero).
El primer factor se denominó "Escala de Respuesta Empática" y su contenido apunta hacia el reconocimiento de respuestas del adulto, atentas y apropiadas a las señales del niño(a), permitiéndole que realice acciones de su interés y, en general, respetando sus iniciativas, su espacio y los objetos que elige para jugar. El adulto es capaz de reconocer las señales del niño(a), interpretarlas adecuadamente y responder 
a sus demandas. Esta escala comprende los indicadores 1, 2, $3,15,17,18,19$ y su estimación de confiabilidad es 0.883 .

El segundo factor se denominó "Escala de Interacción Lúdica", caracterizada por la interacción equilibrada en el juego entre niño(a) y adulto, así como la participación de ambos en forma activa. El adulto es capaz de relacionarse con el niño(a) de manera lúdica, tomando en cuenta sus iniciativas e incorporándolas en el juego. Agrupa los ítems $4,5,9,10,11,13$, con una cofiabilidad de 0.784 .

El último factor se denominó "Escala de Expresión Emocional", representada por un adulto que manifiesta una actitud sensible y cálida frente a las necesidades y emociones del niño(a), a través del lenguaje verbal y gestual, así como a través de las acciones que realiza con el niño(a). Incluye los indicadores $6,7,8,12,14,16$ y tiene una confiabilidad igual a 0.707 .

A partir de estos resultados se construyó el cálculo para el puntaje final de la E.S.A., considerando el puntaje de las tres escalas, ponderándolas según su peso en la varianza de la variable sensibilidad. Así, se llegó a un puntaje final para la E.S.A. entre 1,0 a 3,0, donde mayor puntaje indica una mayor sensibilidad. Finalmente, considerando la distribución de puntajes en la muestra, se calcularon los puntos de corte para asignar las categorías de sensibilidad baja, adecuada y alta.

\section{Discusión y Conclusiones}

El presente estudio se realizó con el objetivo de construir y examinar las propiedades psicométricas de un instrumento para medir la sensibilidad del adulto en su interacción con niños pequeños. Los resultados del estudio preliminar de las propiedades psicométricas evidencian que el E.S.A. es una herramienta que posee un conjunto de reactivos, cuyas rúbricas son suficientemente explícitas, ya que la consistencia entre diferentes observadores en el estudio piloto resulta de muy buen nivel. Asimismo, el análisis de esta muestra proporciona estimadores fiables para evaluar la sensibilidad en adultos y las primeras estimaciones de validez factorial proporcionan un conjunto de tres factores que resultan pertinentes a la medición del constructo.

Los indicadores que se eliminaron de la versión original se referían en su totalidad a indicadores específicos a evaluar en caso que la interacción detallada se diera. Algunos indicadores no fueron observados en ninguno de los casos analizados y en otros su frecuencia era tan baja que no permitía llegar a un acuerdo razonable, por lo cual se optó por eliminarlos.

Los resultados del presente estudio aportan los primeros antecedentes respecto a un nuevo instrumento para medir un constructo de alta significación en psicología, como es la sensibilidad del adulto. De esta manera, permitirá en futuros estudios, contar con una herramienta para investigar las variables asociadas a las competencias involucradas en la sensibilidad de los adultos a cargo de niños pequeños en el contexto chileno, y que pueda a su vez generalizarse posteriormente a contextos latinoamericanos. A la vez, es un instrumento que puede ser utilizado en el contexto de la psicología clínica, al permitir identificar los niveles de sensibilidad de los adultos responsables del niño(a), proporcionando antecedentes acerca del efecto de este atributo referente al adulto sobre el desarrollo emocional de los niños(as).

Por cierto que este estudio es solo la primera aproximación a la medición de un constructo complejo y, por ende, requiere de análisis en otras muestras de mayor tamaño para contrastar si las características encontradas en este trabajo son estables.

\section{Referencias}

Ainsworth, M., Blehar, M., Waters, E., \& Wall, S. (1978). Patterns of Attachment: A psychological study of the strange situation. U.SA.: Lawrence Erlbaum Associates.

Ainsworth, M.D.S., \& Wittig, B.A. (1969). Attachment and exploratory behavior of one-year-olds in a strange situation. In B.M. Foss (Ed.), Determinants of infant behavior (pp. 111 -136). London: Methue.

Atkinson, L., Goldberg, S., Raval, V., Pederson, D., Benoit, D., Moran, G., Poulon, L., London, Myhal, N., Zwiers, M., Gleason, K., \& Leung, E. (2005). On the relation between maternal state of mind and sensitivity in the prediction of infant attachment security. Developmental Psychology, 41, 42-53.

Behrens, K.Y., Parker, A.C., \& Haltigan, J.D. (2011). Maternal sensitivity assessed during the strange situation procedure predicts child's attachment quality and reunion behaviors. Infant Behavior \& Development 34, 378-381.

Belsky, J. (1999). Interactional and contextual determinants of attachment security. In J. Cassidy, \& P. Shaver (Eds.), Handbook of attachment: Theory, Research and clinical applications (pp. 249-264). New York: Guildford Press.

Bornstein, M., Tamis-LeMonda, C., Hahn, Ch., \& Haynes, M. (2008). Maternal responsiveness to young children at three ages: Longitudinal Analysis of a multidimensional, modular, and specific parenting construct. Developmental Psychology in The Public Domain, 44, 867-874.

Bowlby, J. (1969). El vínculo afectivo. Buenos Aires, Argentina: Paidós.

Bowlby, J. (1973). Attachment and loss: Vol. 2. Separation: Anxiety and anger, New York: Basic Books.

Braungart, J., Garwood, M., Powers, B., \& Wang, X. (2001). Parental sensitivity, infant affect, and affect regulation: Predictor of later attachment. Child Development, 72, $252-270$.

Bretherton, I. (2000). Emotional availability: An attachment perspective. Attachment \& Human Development, 2, 233 - 241.

Cassidy, J., \& Shaver, P. (1999). Hanbook of attachment. Theory, research, and clinical aplications. London: The Guildford Press.

Crittenden, P. (2005). Care-Index para toddlers: Manual de Codificación. Miami, EEUU: Family Relations Institute.

Crittenden, P. (2006). A dynamic maturational model of attachment. ANZJFT, 27, 105-115.

Cox, M.J., Owen, M.T., Henderson, V.K., \& Margand, N.A. (1992), Prediction of infant-father and infant-mother attachment. Development Psychology, 28, 474-483.

Hirsh-Pasek, K. \& Burchinal, M. (2006). Mother and caregiver sensitivity over time: Predicting language and academic outcomes with variable- and person-centered approaches. Merrill-Palmer Quarterly, $52,449-485$.

Isabella, R.A. (1993). Origins of attachment: Maternal interactive behavior across the first year. Child Development, 64, 605-621. 
Isabella, R. A., \& Belsky, J. (1991) interactional synchrony and the origins of infant-mother attachment. Child Development, 62, 373-384.

Jin, M. K., Jacobvitz, D., Hazen, N., \& Jung, S. H. (2012). Maternal sensitivity and infant attachment security in Korea: Cross-cultural validation of the Strange Situation. Attachment \& Human Development. 14, 33-44.

Lamb, M. E., Thompson, R. A., Gardner, W. P., Charnov, E. L., \& Estes, D. (1984). Security of infantil attachment as assessed in the "strange situation": Its study and biological interpretation. The Behavioral and Brain Sciences, 7, $127-171$.

Leerkes, E. M., Parade, S. H., \& Gudmundson, J. A. (2011). Mothers emotional reactions to crying pose risk for subsequent attachment insecurity. Journal of Family Psychology. 25, 635-643.

Leigh, P. (2011). Maternal sensitivity and language in early childhood: A test of the transactional model. Perceptual and Motor Skills. 113, 281-299.

Lohaus, A., Keller, H., Ball, J., Elben, C., \& Voeker, S. (2001). Maternal sensitivity: Components and relations to warmth and contingency. Parenting, 1, $267-284$.

Lok, S., \& McMahon, C. (2006). Mothers thoughts about their children: Links between mind mindedness and emotional availability. British Journal Of Development Psychology, 24, 447-488.

Meins, E., Fernyhough, Ch., Fradley, E., \& Tuckey, M. (2001). Rethinking maternal sensitivity: Mothers' comments on infants' mental Processes Predict Security of Attachment at 12 Months. Journal of Child Psychology and Psychiatry. 42, 637-648.

Mileva-Seitz,V., Kennedy, J., Atkinson, L., Steiner, M., Levitan, R., Matthews, S. G., Maney, M. J., Sokolowski, M. B., \& Fleming, A.S. (2011). Serotonin transporter allelic variation in mothers predicts maternal sensitivity, behavior and attitudes toward 6-month-old infants. Genes, Brain and Behavior, 10, 325-333.

Mills-Koonce, W. R., Gariépy, J. L., Propper, C., Sutton, K., Calkins, S., Moore, G., \& Cox, M. (2007). Infant and parent factors associated with early maternal sensitivity: A caregiver-attachment systems approach. Infant Behavior \& Development, 30, 114-126.

Mills-Koonce, W. R., Appleyard, K., Barnett, M., Deng, M., Putallaz, M., \& Cox, M. (2011). Adult attachment style and stress as risk fac- tors for early maternal sensitivity and negativity. Infant Mental Health Journal, 32, 277-285.

NICHD Early Child Care Research Network. (1997). The effects of infant child care on infant-mother attachment securing: Results of the NICHD study of early child care. Child Development, 68, 860-879.

NICHD, Early Child Care Research Network (2006). Child-Care effect sizes for the NICHD study of early child care youth development. American Psychologist, 61, 99 - 116.

Pederson, D., Moran, G., Sitko C., Campbell, K., Ghesquire, K., \& Acton, H. (1990). Maternal sensitivity and the security of infant-mother attachment: A Q-Sort Study. Child Development, 61, 1974-1983.

Pederson, D., \& Moran, G. (1995). A categorical description of attachment relationships in the mode and its relation to q-sort measures of infant-mother interaction. In E. Waters, B. Vaughn, G. Posada, \& K. Kondo-Ikemura (Eds.), Caregiving, cultural and cognitive perspectives on secure-base behavior and working models: New growing points of attachment theory and research, Monographs of the Society for Research in Child Development (pp. 247-254), 2-3, Serial No. 244. Washington, D.C.: The Society.

Prior, V., \& Glaser, D. (2006). Understanding attachment and attachment disorders: Theory, evidence and practice. London: Jessica Kingsley Publishers.

Symons, D., \& Clark, S. (2000). A longitudinal study of mother-child relationships and theory of mind in the preschool period. Social Development, 9, 1-23.

Van IJzendoorn, M. (1995). Adult attachment representations, parental responsiveness, and infant attachment: A meta-analysis on the predictive validity of the adult attachment interview. Psychological Bulletin, 117, 387-403.

Van den Boom, C. (1997). Sensitivity and attachment: Next steps for developmentalists. Child Development, 64, 592-592.

West, J. (2000). Terapia de juego centrado en el niño. México: El Manual Moderno. 
